\title{
ECOPED: An Informative System for Pediatric Echocardiography
}

\author{
S Dalmiani ${ }^{1}$, P Festa $^{2}$, L Ait Ali ${ }^{3}$, A Vellani $^{1}$, A Taddei $^{1}$ \\ ${ }^{1}$ Institute of Clinical Physiology - CNR, Pisa, Italy \\ 2“G Pasquinucci” Hospital, Massa, Italy \\ ${ }^{3}$ The Sant'Anna School of Advanced Studies, Pisa, Italy
}

\begin{abstract}
Diagnosis of congenital heart disease in young patients represents a complex assessment of instrumental parameters and requires often a surgical procedure to correct structures morphologies and related functions.

An information system for the management of echocardiographic examinations in patients with suspected or ascertained congenital heart disease, was developed in our departments of Pediatric Cardiology and Cardiac Surgery. This system uses a new model for workflow and clinical dataset collection.

For each diagnostic related group, according to EACTS Congenital Database and European Pediatric Cardiology nomenclatures, was defined a set of relevant echocardiographic data to be collected (Pathology Related Dataset, PRD), in order to comply with a comprehensive diagnostic assessment of the patient.
\end{abstract}

\section{Introduction}

Echocardiography represents a mandatory examination in the process of clinical care for pediatric cardiology, and each patient is evaluated in the Echocardiographic Laboratory for diagnosis and care delivery management.

Structured data collection of Echo exams is mandatory for a complete diagnostic assessment and reporting, and uniformity of parameter collection is relevant for followup and research purposes. Furthermore several measurement, expressed as deviation from normal indexed values (z-value), are of paramount importance in clinical decision process.

In this contest we should consider even interaction among specialists, where a cardiologist and a cardiac surgeon often collaborate for patient care; in this interaction a procedure planned by surgeons needs some morphological parameter that should be collected by cardiologists. In these circumstances, structured data collection should include even parameters procedure oriented, other than other cardiologist's items, and defined datasets represent a "common language" for patient care.
At present, the technological scenario is characterized by a minor percentage of computerized systems which aid clinicians to obtain a proper assessment for patients, from newborns to Grown-Up Childs (GUCH).

Aim of this project was to develop a standard diagnosis-related echocardiographic dataset, supported by an informative system capable to manage echo examinations in patients with congenital heart disease

\section{Methods}

After evaluation of clinicians needs, was considered to create a dataset related to each diagnosis. From an exhaustive internal classification of 355 congenital heart disease, including pre- and post-procedure status, a correspondence with EACTS Congenital Database and European Pediatric Cardiology nomenclatures were developed, to gain an higher level of relationship with international initiatives.

A list of main diagnosis is shown in Table 1.

Each dataset, called Pathology-Related Dataset (PRD) due to its relationship to a pathology, includes a set of relevant data selected in order to comply with a comprehensive diagnostic assessment, according to the known guidelines and possible related surgical procedure.

Each PRD item is defined as:

- coded values, for assessment of diagnosis, morphological structure, functional evaluation, etc.

- numeric values, for measurement

- free text, when a consistent codification is inapplicable.

Where a single item can be assigned to many PRD (e.g. age, gender, \%EF, body surface area, etc).

Many assessment are composed of associated pathology, not always a principal and other secondary but some more or less of the same importance; in this case intersection of items collected in a previous PRD was decided to be automatically reported with entered value, and enhanced with the pathology specific items (Figure 1). 


\begin{tabular}{|c|c|c|c|}
\hline Normal & $\begin{array}{l}\text { Position/laterality } \\
\text { anomalies }\end{array}$ & $\begin{array}{l}\text { Systemic venous return } \\
\text { anomalies }\end{array}$ & $\begin{array}{l}\text { Pulmonary venous return } \\
\text { anomalies }\end{array}$ \\
\hline Cardiac septation defects & $\begin{array}{ll}\text { Right } & \text { Heart } \\
\text { malformations } & \end{array}$ & Left Heart malformations & $\begin{array}{l}\text { Atrio-Ventricular/ } \\
\text { Ventriculo-arterial } \\
\text { connection anomalies }\end{array}$ \\
\hline Cardiomyopathy & Endocardits & Intracardiac mass & $\begin{array}{l}\text { Pericardial/ Pleural Effusion / } \\
\text { Diapragm anomalies }\end{array}$ \\
\hline Post surgical repair & $\begin{array}{ll}\begin{array}{l}\text { Post } \\
\text { procedure }\end{array} & \text { interventional } \\
\end{array}$ & & \\
\hline
\end{tabular}

Table 1: principal node of diagnosis classification

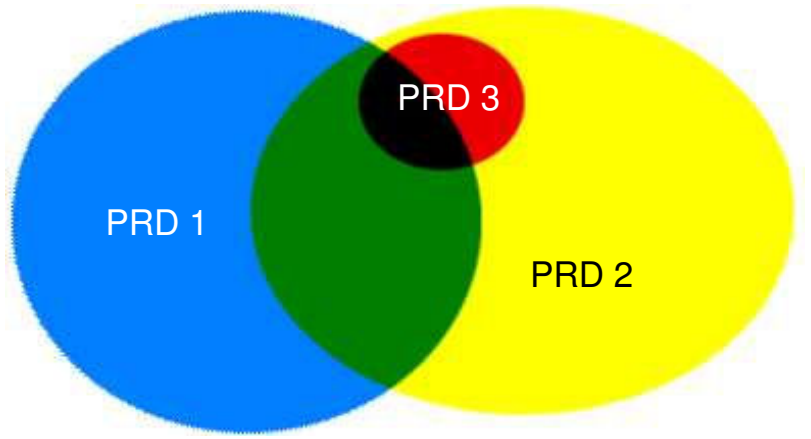

Figure 1: Intersection of PRD is a common feature of developed approach

For main anatomical structures, indexed normal values related to patient grown was noticed to be of foremost importance.

Relevant measurements of anatomic structures were associated with an on-line calculation of normal value and standard deviation (Z-value) subject parameter related to body surface area, age and gender [1, 2, 3, 4, 5].

To solve generic problem of exam reporting, after structured data collection, was decided to generate automatically a final report, comprehensive of demographic data, vital parameters and containing only the collected data, together with conclusive physician's comments.

To guarantee an effective test site, the informative system has been implemented on a network environment, and developed in a real clinical environment with Pediatric Cardiology and Cardiac Surgery departments at our institute (Figure 2).

The clinical information system contributes to integrate patient data collected by different sources of information, represented by visits, tests and examinations performed within specific diagnostic laboratories. These sub-systems, called "Functional Islands", other than managing exams performing, gather data from their internal archives and send them to a central repository, which duty is the integration of all information concerning the single patient to set up the overall EMR view $[6,7]$.

To obtain a friendly and flexible system and to follow quickly last-minute changes on dataset specifications, an XP development approach was adopted.

Extreme Programming (XP) [8] is a software development approach that represents an effective method for building smaller systems in an environment where requirements are changing continuously.

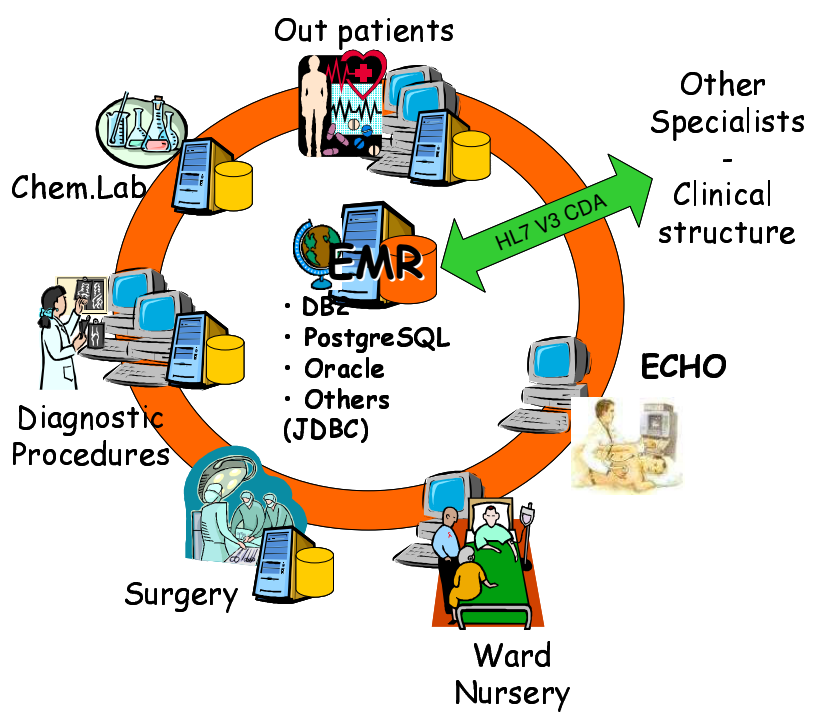

Figure 2: Functional architecture of Hospital Information System where ECHOPED has been integrated

In project development we faced heterogeneous types of workstation, using different operating systems. On the 
other hands, one of the most common requirements for new software or systems is the capability to run on different machines and under different operating systems. In order to comply with these constraints, our ECHOPED system was developed in Java language, which guarantees a free integration on many popular operating systems and platforms and allows, through the JDBC protocol, an easy integration with many different database systems.

\section{Results}

Each determined PRD was documented and an informative system was implemented, where data collection is supported by an ergonomic graphical interface that allows rapid data entry and a full integration with Hospital Information Systems (HIS) (Figure 3-4).

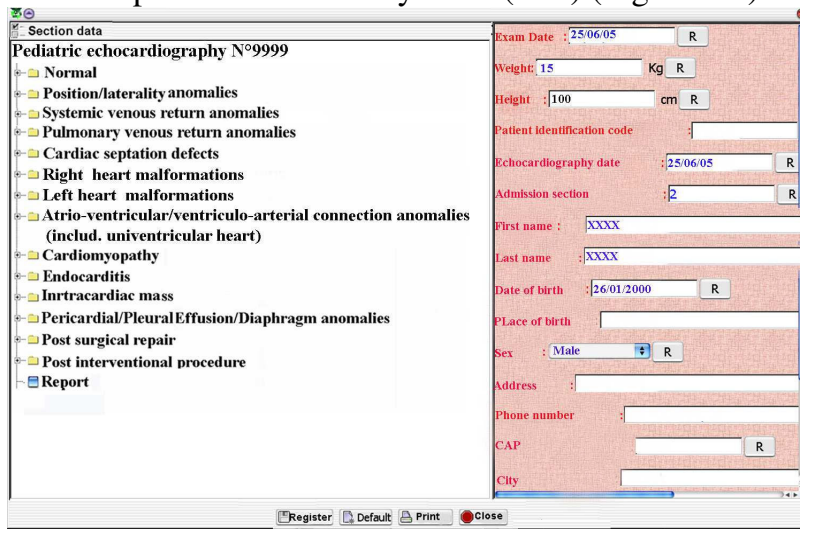

Figure 3: Graphical interface representing main diagnosis classification

Underlying storing system $[9,10]$ is able to recognize the same definition entered in previous PRD, and repropose already entered values.

Hypothetic or conclusive Diagnosis are represented graphically as a tree structure, where the underlying graph structure has been exploited with repetition of the multiple connected PRD in many branch. The tree nodes represents a diagnostic hint, with an associated PRD that allow a more specific assessment of pathology..

The tree leaves represents a specific diagnosis, and many leaves can be selected, in order to document a complex multiple diagnosis.

Major advantages of this system are:

- $\quad$ get uniformity of measurement for each pathology or surgical procedure;

- get uniformity of diagnosis chosen in connection with ECSUR/EACTS list;

- $\quad$ obtain a comprehensive diagnostic data base of all patients undergoing echo examination;

- record, archive and compare the measurements along different echo exams in the same patient;

- $\quad$ get on-line indexed normal values and $\mathrm{z}$-values for the main measurements;

- $\quad$ make the echo report faster (Figure 5);

- obtain a teaching instrument for fellows or technicians performing echocardiographic exams in congenital heart disease patients.

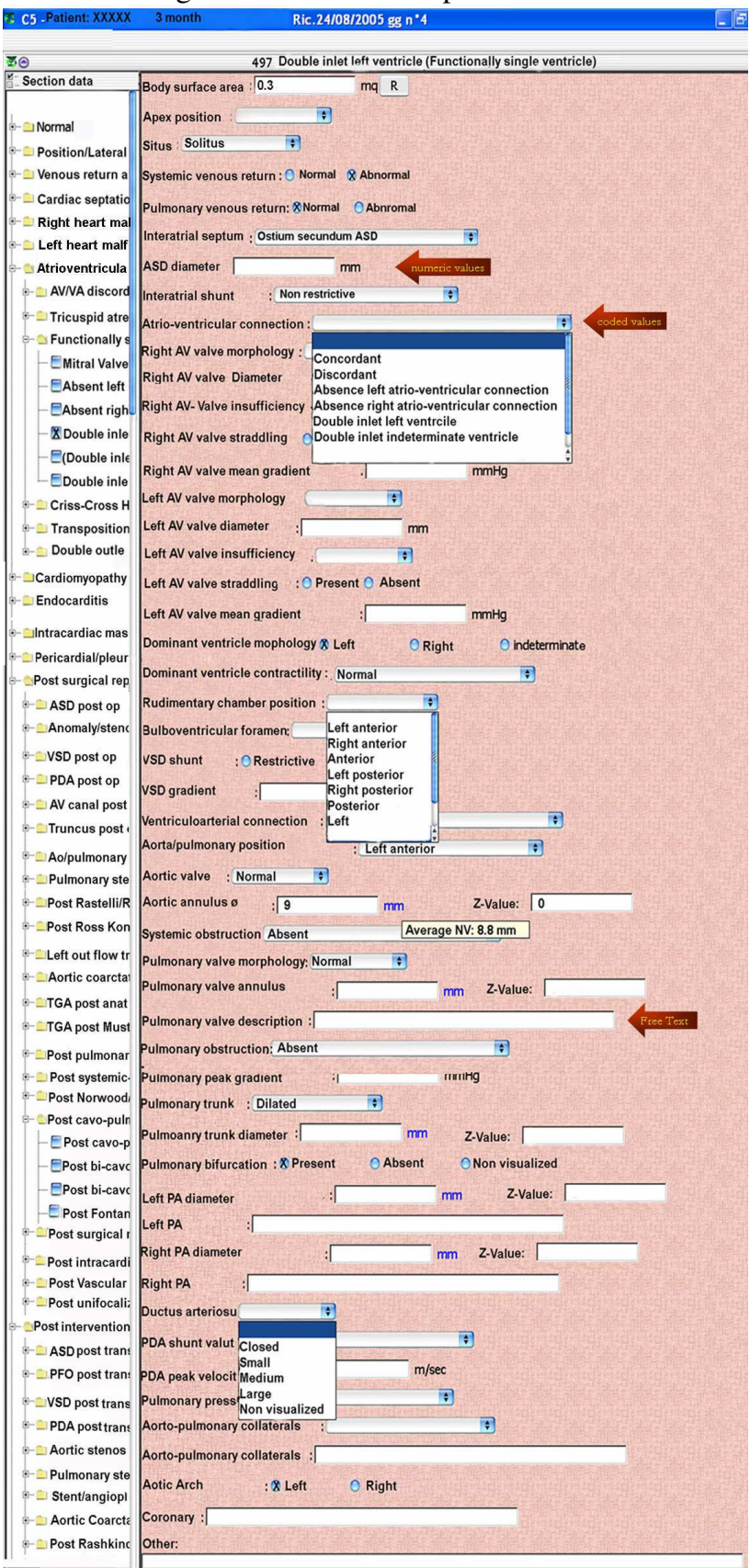

Figure 4: sample of graphical interface for double inlet left ventricle 
Developed system was routinely used since March 2005; until September 2005900 Echocardiographic examinations have been performed with this auxiliary system.

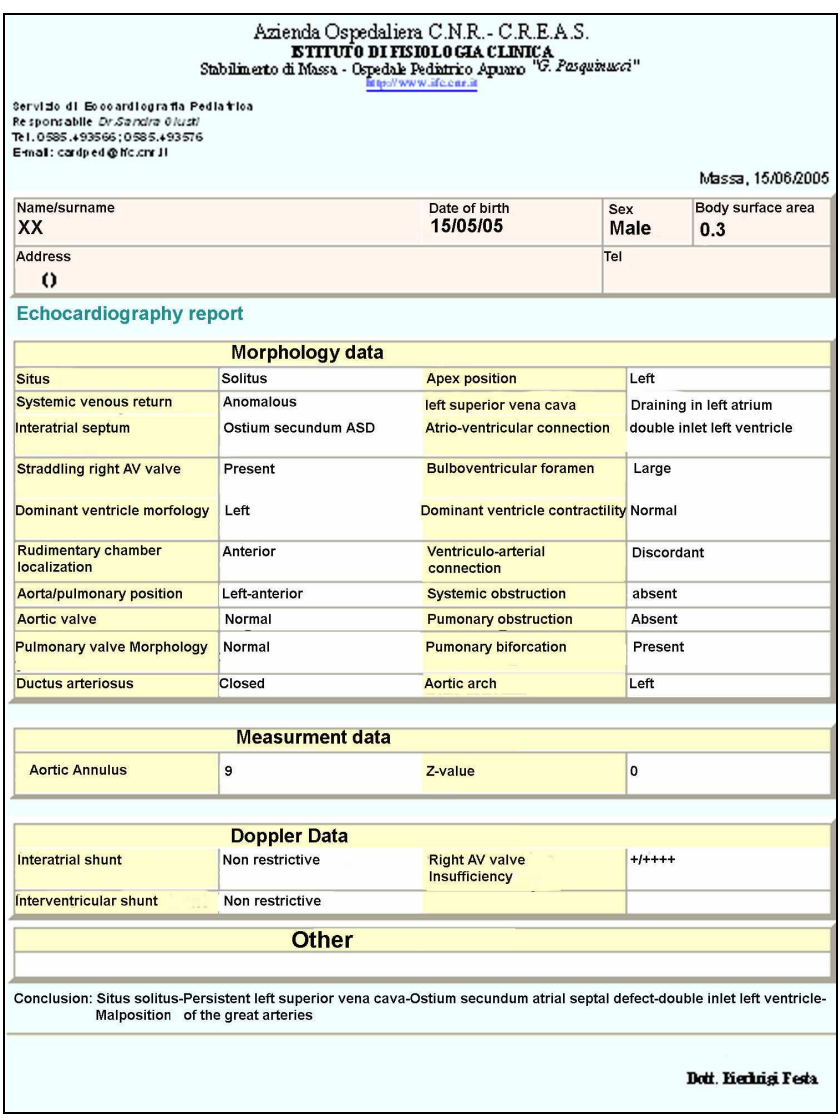

Figure 5: Generated text report of executed exam, with collected parameters and final conclusions

\section{Discussion and conclusions}

This system uses a new definition of workflow and clinical dataset collection, and have been thoroughly experimented in our O.U.

This Informative System for Pediatric Echocardiography allow us to:

- obtain a comprehensive diagnostic database of all patients evaluated with echo examinations in our hospital;

- $\quad$ standardize measured parameters for each pathology or related intervention;
- record and compare the measurements along different echo exams in the same patient;

- get indexed normal values and $\mathrm{z}$-values for main measurements;

- $\quad$ speed-up echo reporting;

- train fellows and technicians in pediatric echocardiography.

\section{References}

[1] Relationship of the dimension of cardiac structures to body size: an echocardiographic study in normal infants and children, In Cardiol. Young 1999;9;402-410

[2] Two-Dimensional Echocardiographic Valve Measurements in Healthy Children: Gender Specific Differences, In Pediatric Cardiology 2004, 10.1007; Springer-Verlag

[3] Echocardiographic Assessment of Aortic Root Dimensions in Normal Children Based on Measurement of a New Ratio of Aortic Size Independent of Growth, In America Journal of Cardiology 1995;75;711-715

[4] Limits of Normal Left Ventricular Dimensions in Growth and Development: Analysis of Dimensions and Variance in the Two-Dimensional Echocardiograms of 268 Normal Health Subjects, In Journal American College Cardiologists 1988;12;1432-1441

[5] Two-Dimensional Echocardiographic Determination of Aortic and Pulmonary Artery Sizes from Infancy to Adulthood in Normal Subjects, In America Journal of Cardiology 1984;53;218-224

[6] Macerata A, Landucci L, Pierotti D et al. Networking for health care administration and delivery: the information system at the CNR Institute of Clinical Physiology. In: Proceedings of Health Telematics '95.Pisa: 1995, 113-18.

[7] Morales MA, Dalmiani S, Carpeggiani C et al.: Electronic Medical Records in a Cardiological Outpatient Clinic In: Computers in Cardiology 2002; 29: 381-84

[8] Beck K. Extreme Programming Explained: Embrace Change. Addison-Wesley, 1999; ISBN 201-61641-6.

[9] Taddei A, Carpeggiani C, Emdin M et al: Development of an electronic medical record for patient care in Cardiology. In: Computers in Cardiology 1997; 22: 641-44.

[10] An Informative System for Structured Data Management to Build a Cardiological Multidimensional Database, In Computers in Cardiology 2002; 29: 369-372

Address for correspondence

Stefano Dalmiani, PhD.

CNR Institute of Clinical Physiology

Via Giuseppe Moruzzi 1

I-56124 Pisa, Italy

Phone: +390503152427

Fax: $\quad+390503152311$

E-mail: dalmiani@ifc.cnr.it 